\title{
A systematic review of factors affecting quality of life after postmastectomy breast reconstruction in women with breast cancer
}

\author{
Helena Sousa $^{1}$ | Sónia Castro ${ }^{2}$ | Joaquim Abreu ${ }^{3}$ | M. Graça Pereira ${ }^{1,4}$ (C)
}

\footnotetext{
${ }^{1}$ Family Health and IIIness Research Group, School of Psychology, University of Minho, Braga, Portugal

${ }^{2}$ Breast Cancer Clinic/Psycho-Oncology Service, Francisco Gentil Portuguese Institute of Oncology of Porto, Porto, Portugal

${ }^{3}$ Breast Cancer Clinic/Head of the Surgical Oncology Department, Francisco Gentil Portuguese Institute for Oncology of Porto, Porto, Portugal

${ }^{4}$ Research Center in Psychology (CIPsi), School of Psychology, University of Minho, Braga, Portugal

Correspondence

M. Graça Pereira, Associate Professor with Aggregation, School of Psychology, University of Minho, Campus de Gualtar, 4710 Braga, Portugal.

Email: gracep@psi.uminho.pt
}

\begin{abstract}
Objective: The aim of this study is to present a broader perspective of factors affecting the quality of life after postmastectomy breast reconstruction in women with breast cancer by considering these patients' self-reported outcomes.

Methods: The search was performed from 29 March to 19 April 2019, on the following databases: Psyclnfo; Web of Science Core Collection, Current Contents Connect, Derwent Innovations Index, $\mathrm{KCl}$-Korean Journal Database, Russian Science Citation Index, SciELO Citation Index, and MEDLINE. The studies were included if they identified factors affecting self-reported quality of life after breast reconstruction, in women with breast cancer.

Results: One hundred and twenty-two records were identified. After quality assessment, 44 studies were included for qualitative synthesis. This review comprised a total of 16683 women who underwent breast reconstruction. The results identified a broad collection of 32 empirically based variables associated with several domains of quality of life. These variables were grouped into four categories: (a) surgical, (b) clinical, (c) psychosocial, and (d) sociodemographic variables.

Conclusions: This systematic review goes beyond surgical factors of morbidity and uses patient-reported outcomes to scope wider issues that influence quality of life, such as psychosocial and sociodemographic variables. It sustains the need to adopt a more holistic approach and advises the consideration of preoperative psychosocial factors to better understand these patients' quality of life after breast reconstruction and to implement future preventive measures.
\end{abstract}

\section{KEYWORDS}

breast cancer, breast reconstruction, cancer, oncology, patient-reported outcomes, quality of life

\section{1 | INTRODUCTION}

Previous research has sustained the important role of breast reconstruction after breast cancer mastectomy. ${ }^{1}$ However, recent studies suggested that quality of life improvements after this procedure are not as expected since, for some patients, quality of life after breast reconstruction was unchanged, ${ }^{2,3}$ worse, ${ }^{4-6}$ or similar to the quality of life of women with mastectomy alone. ${ }^{4,7-9}$

Understanding which factors are more likely to affect the quality of life after this procedure is of great clinical importance to help prevent postreconstruction adjustment problems and decisional regret. ${ }^{10}$ Previous reviews discussed the role of surgical variables as possible risk 
factors. ${ }^{11,12}$ Results revealed similar outcomes for immediate and delayed procedures, except for sexual function favoring immediate reconstruction. ${ }^{13,14}$ Autologous reconstruction was also related to higher levels of psychosocial and sexual well-being, compared with implant-based procedures. ${ }^{15}$ The integration of postreconstruction radiation has also been suggested since the overall quality of life, evaluated from the patient's perspective, does not seem to be compromised. ${ }^{16,17}$ However, the assessment of surgical-related variables is no longer sufficient to understand the multidimensional construct of quality of life which refers to a combination of physical, psychological, social, and spiritual domains. ${ }^{18,19}$ According to the World Health Organization, quality of life can be defined as the perception of individuals about their place in the culture and value system in relation to their aims, expectations, standards, and concerns. ${ }^{20}$

Considering the definition of quality of life, and that the ultimate goal of breast reconstruction is the improvement of quality of life, the patients' perception must be considered. ${ }^{15}$ However, few patient characteristics have been discussed in the literature, such as breast characteristics, age, and comorbidities. ${ }^{21}$ One narrative review pointed to the importance of exploring the patients' unrealistic expectations towards this procedure, suggesting that patient education could improve quality of life outcomes. ${ }^{22}$ To our knowledge, only one integrative review discussed a broader range of variables that have been found to influence patient satisfaction, body image and quality of life in this population, suggesting premorbid influences, disease, and treatment-related factors as possible risk factors. ${ }^{23}$

Therefore, the aim of this study is to present a broader perspective of factors affecting quality of life after breast reconstruction in women with breast cancer by considering studies which included patients' self-reported outcomes.

\section{2 | METHODS}

This review was conducted following the principles of a systematic search. $^{24,25}$ For a more detailed presentation of all of the PRISMA 2009 checklist items, see Appendix 1 (section A).

\section{1 | Eligibility criteria}

Studies were included if the sample consisted of women with breast cancer exposed to postmastectomy breast reconstruction. Exclusions were made when the sample included only patients with prophylactic reconstructions due to gene mutation, if patient-reported outcome measures (PROMs) of quality of life after breast reconstruction were not assessed, and if they did not identify factors affecting selfreported quality of life.

\section{2 | Search}

Studies were identified by searching Psyclnfo and Web of Science Core Collection, Current Contents Connect, Derwent Innovations
Index, $\mathrm{KCl}$-Korean Journal Database, Russian Science Citation Index, SciELO Citation Index, and MEDLINE. The search was performed by one author (HS) from 29 March to 19 April 2019.

For more information regarding the full electronic search strategy and the keywords applied, see Appendix 1 (section B).

\section{3 | Study selection}

Eligibility assessment was performed independently by two authors (H.S. and S.C.), with an interrater agreement of $97.7 \%$. Discrepancies were resolved by discussion and consensus. Study selection ended with 44 full-text articles.

\section{4 | Data collection}

The data collection process was performed independently by one author (H.S.) and confirmed by a second author (S.C.). Data were collected for the following items: studies characteristics (date and design), sample characteristics (size, country, age, type and timing of reconstruction, and adjuvant treatments), and outcome variables (quality of life tools, factors affecting quality of life, statistics used, and major results).

\section{5 | Critical appraisal}

Critical appraisal was performed with the Joanna Briggs Institute Checklists for Cross-sectional and for Cohort Studies. These checklists aim to assess the methodological quality of a study regarding the possibility of bias in its design, conduct, and analysis.

\section{3 | RESULTS}

\section{1 | Study selection}

Figure 1 presents the PRISMA flow diagram with the different stages of study selection.

\subsection{Studies characteristics}

The 44 included papers were published from 2000 to 2018. Most studies had a retrospective cross-sectional design $(n=31), 12$ studies were prospective, and one was ambispective. All of the included studies performed quantitative analysis.

\section{3 | Critical appraisal}

Two reviewers conducted the appraisal and its ratification (H.S. and S. C.) with an interrater agreement of $93.2 \%$. Discrepancies were solved by discussion and consensus. Studies were rated as good $(n=16)$, fair ( $n=16$ ), poor ( $n=9)$, and very poor $(n=3)$ regarding their quality. Most studies relied on medical records to assess clinical variables, which may introduce some bias as reliability was not assured. Most studies $(56.8 \%)$ did not adjust for possible confounding factors, which 
FIGURE 1 PRISMA flow diagram with the different stages of study selection

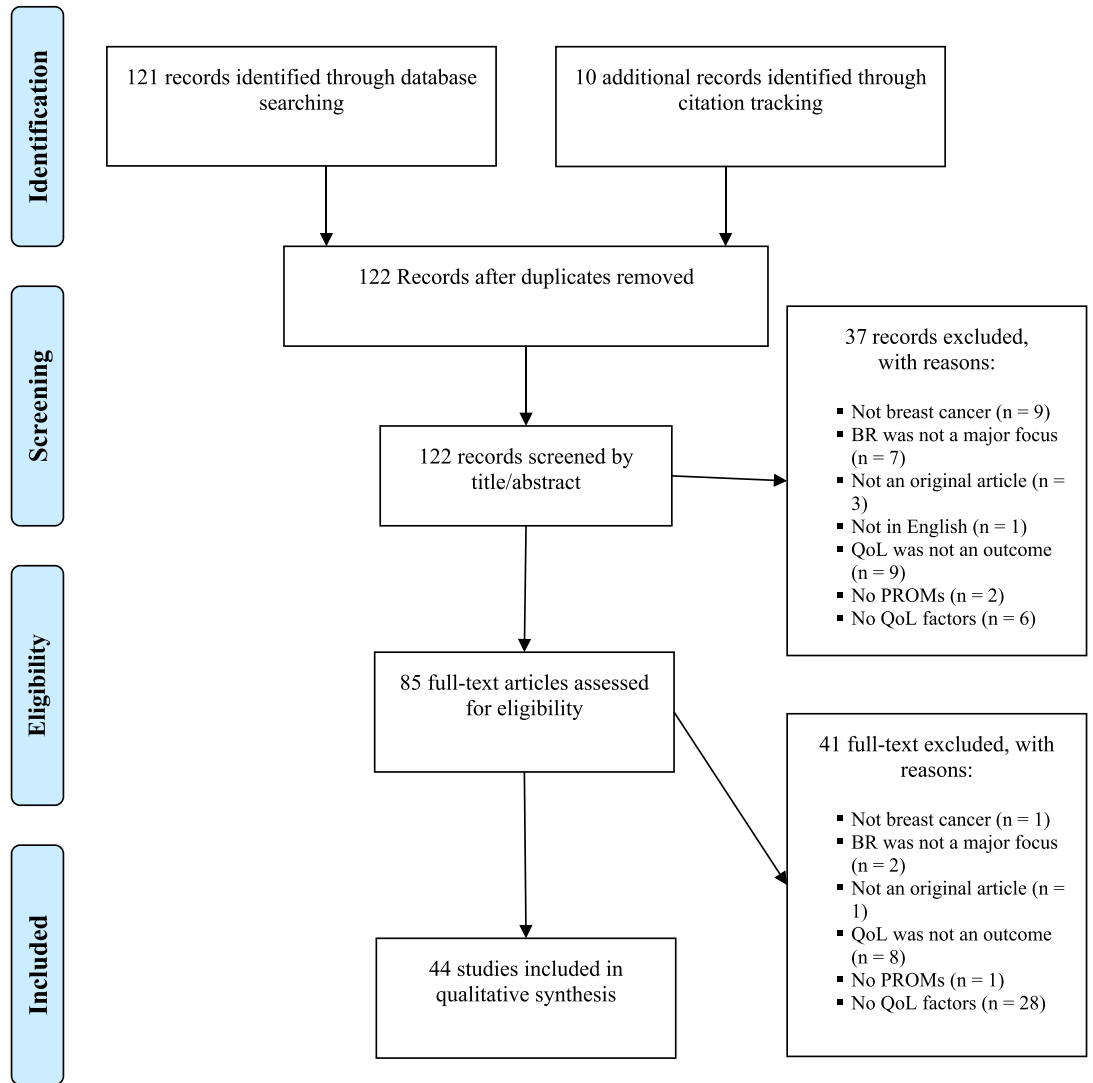

For supporting information on these measures, see Appendix 2 (Section B).

From preconstruction to postreconstruction, five studies reported overall improvement, ${ }^{14,27-30}$ while four studies failed to sustain these findings. ${ }^{3,31-33}$ Three studies reported decreased physical and social functionality. $5,6,34$

\subsection{Factors affecting quality of life after breast reconstruction}

Of the 44 included studies, 29 variables presented statistically significant associations with quality of life dimensions $(P<.05)$. Effect sizes (Cohen's $d$ and $f$ ) were calculated for all outcomes if the necessary information was provided by the included studies. Other analyses have resulted in the transformation of these statistics into odds ratio (OR), to help compare the magnitude of the different variables in the main outcome. Next, three nonstatistically significant variables were included for qualitative analysis, since they presented medium to large OR. $^{35}$

Table 1 presents all of the 32 factors affecting quality of life after breast reconstruction and the number of appearances across studies. Two authors (H.S. and S.C.) independently grouped each of these variables into the following categories: (a) surgical-related variables, (b) patients' clinical characteristics, (c) psychosocial variables, and (d) patients' sociodemographic characteristics. Inter-rater reliability resulted in a $96 \%$ agreement between raters. Disagreements were discussed, and a third author was consulted (M.G.P.). applied. The most commonly used was the BREAST-Q $(n=25)$. 
TABLE 1 Factors affecting quality of life after postmastectomy breast reconstruction in breast cancer women, grouped by category type. Number of appearances across studies are also indicated

\begin{tabular}{|c|c|c|c|}
\hline Surgical-related variables $(n=9)$ & Clinical variables $(n=9)$ & Psychosocial variables $(n=9)$ & Sociodemographic variables $(n=5)$ \\
\hline Type of reconstruction $(n=20)^{a}$ & Radiotherapy $(\mathrm{n}=13)^{\mathrm{a}}$ & Prereconstruction QoL $(n=3)^{a}$ & Age $(n=14)^{a}$ \\
\hline Timing of reconstruction $(n=11)^{a}$ & Chemotherapy $(n=6)^{a}$ & Depression $(n=2)^{a}$ & Marital status $(n=4)^{a}$ \\
\hline Type of mastectomy $(n=4)^{a}$ & Cancer stage $(n=4)^{a}$ & Satisfaction with esthetic outcome $(n=2)^{a}$ & Work status $(n=4)^{a}$ \\
\hline Type of implant $(n=4)^{a}$ & Smoking status $(\mathrm{n}=2)^{\mathrm{a}}$ & Decision-making processes $(n=1)^{a}$ & \multirow[t]{4}{*}{ Race $(n=2)^{a}$} \\
\hline Time after reconstruction $(n=2)^{a}$ & Immunotherapy $(\mathrm{n}=1)^{\mathrm{a}}$ & Psychosocial well-being $(n=1)^{a}$ & \\
\hline Previous breast surgery $(n=1)^{a}$ & Anxiolytic therapy $(n=1)^{b}$ & Sexual well-being $(n=1)^{a}$ & \\
\hline Breast symmetrization $(n=1)^{b}$ & Lymphedema $(n=1)^{a}$ & Interpersonal problems $(\mathrm{n}=1)^{\mathrm{a}}$ & \\
\hline
\end{tabular}

${ }^{a}$ Variables that reached statistical significance $(P<.05)$ at least once, on the included studies.

${ }^{b}$ Variables that did not reach statistical significance $(P<.05)$ but had medium or large effect sizes (odds ratio $\geq 2$, according to Sullivan and Feinn's editorial review from 2012).

Table 2 shows all the main results of the 44 included studies for qualitative synthesis. Only medium to large OR were reported. For support information on the calculation of effect sizes, see Appendix 3.

\section{7 | Surgical-related variables}

Type of mastectomy, previous breast surgery, breast symmetrization, timing of breast reconstruction, type of breast reconstruction, time after breast reconstruction, type of implants, fat-grafting, and complications were identified as variables affecting patient-reported outcomes of quality of life.

Regarding the type of mastectomy, three studies out of four (75\%) found that patients who underwent nipple-sparing mastectomy reported significantly higher psychosocial ${ }^{36}$ and sexual well-being, ${ }^{37}$ when compared with non-nipple-sparing surgeries. Skin sparing mastectomy has also been found to better preserve physical well-being (in the chest), compared with modified radical mastectomy. ${ }^{10}$

Six studies out of 11 (54\%) reported no significant differences between immediate and delayed procedures. ${ }^{14,26,32,34,38,39}$ Studies that identified between group differences also reported mixed findings. On one hand, two studies reported the benefits of a delayed procedure for emotional, ${ }^{40}$ psychosocial, sexual, and physical wellbeing, ${ }^{30}$ while, on the other hand, three studies reported the benefits of an immediate breast reconstruction for physical, sexual, and emotional well-being. ${ }^{41-43}$

Nine studies out of 20 (45\%) reported results favoring autologous procedures for psychosocial and sexual well-being. 5,12,15,30,37,40,43-45 Some studies also differentiated between different types of autologous procedures, favoring DIEP flaps over TRAM for psychosocial and physical well-being on the abdomen. ${ }^{42,45}$ No significant differences were found between patients who underwent TRAM or latissimus dorsi flap. ${ }^{3,13,28}$ One study found that implant-related breast reconstruction resulted on better physical function on the chest compared with autologous tissue reconstructions, which, in spite of its benefits regarding psychosocial and sexual function, seemed to be more associated with lower vitality, ${ }^{46}$ and higher body pain ${ }^{5,6}$ than implant-based reconstructions.

Results regarding the association of fat grafting procedures with quality of life are mixed, since one study reported no significant associations, ${ }^{47}$ while the other study reported fat grafting to be associated with psychosocial and sexual well-being. ${ }^{29}$

Regarding the choice between saline or silicone implants, the results pointed towards higher physical, psychosocial, and sexual well-being after silicone implant. ${ }^{48}$ One study also suggested that there were no significant differences regarding the shape of the implant. ${ }^{49}$

Two studies assessed the role of time after reconstruction on these patients' quality of life. Santosa et al, ${ }^{37}$ found that psychosocial, physical, and sexual well-being increased with time after this surgery. However, Matthews and colleagues ${ }^{50}$ did not find this variable to be a significant predictor of quality of life.

Lastly, four studies out of 11 (36\%) suggested that complications had a significant impact on physical, emotional, and sexual wellbeing. ${ }^{6,15,40,41}$ Menez et $\mathrm{al}^{41}$ reported that major complications (vs minor) were associated with lower psychosocial, emotional, and sexual well-being, while Winters et $\mathrm{al}^{6}$ found that early complications (vs later) were associated with lower overall quality of life.

\section{8 | Patients clinical characteristics}

This review found important results regarding the associations between quality of life dimensions and clinical variables such as radiotherapy, chemotherapy, immunotherapy, cancer stage, lymphedema, body mass index (BMI), smoking status, and taking anxiolytics.

Three studies out of $13(23 \%)$ reported significant associations between radiotherapy and lower social function, ${ }^{6}$ lower physical well-being on the chest, ${ }^{51}$ and lower psychosocial, physical, and sexual well-being. ${ }^{37}$ Most studies reported nonstatistically significant results with low effect sizes. 
TABLE 2 Factors affecting quality of life after breast reconstructions, major results and effect sizes

\begin{tabular}{|c|c|c|c|c|}
\hline $\begin{array}{l}\text { Critical } \\
\text { Appraisal }\end{array}$ & References & QoL Tools & Factors Affecting QoL & Results \\
\hline \multirow[t]{18}{*}{ Good $(N=16)$} & $\begin{array}{l}\text { Bennet et al, } \\
2017\end{array}$ & BREAST-Q & Fat grafting & $\begin{array}{l}\text {-Fat grafting } \downarrow \text { psychosocial and sexual well- } \\
\text { being }\end{array}$ \\
\hline & $\begin{array}{l}\text { Beugels et al, } \\
2018\end{array}$ & BREAST-Q & Timing of reconstruction (IR vs DL) & -NS \\
\hline & $\begin{array}{l}\text { Dauplat et al, } \\
2017\end{array}$ & QLQ-C30; BR23 & $\begin{array}{l}\text { Age at interview; BMI; work status; cancer stage; } \\
\text { satisfaction with outcome }\end{array}$ & $\begin{array}{l}\text {-Younger patients }(<60 \mathrm{y}) \uparrow \mathrm{QoL} \\
-\downarrow \mathrm{BMI} \uparrow \mathrm{QoL} \\
\text {-Active work } \uparrow \mathrm{QoL} \\
\text {-Advanced stages } \downarrow \text { QoL } \\
\text {-Satisfaction } \uparrow \mathrm{QoL}\end{array}$ \\
\hline & $\begin{array}{l}\text { Eltahir et al, } \\
2015\end{array}$ & BREAST-Q & Type of reconstruction (implant vs FLAP) & -NS \\
\hline & $\begin{array}{l}\text { Eriksson et al, } \\
2013\end{array}$ & BREAST-Q & $\begin{array}{l}\text { RT; neo-CT; BMI; age at interview; type of } \\
\text { implant; presence of a plastic surgeon at MT; } \\
\text { plastic surgeon experience }\end{array}$ & $\begin{array}{l}\text {-For psychosocial well-being: no } \mathrm{RT}>\text { prior } \\
\text { to } \mathrm{BR}(\mathrm{OR}=3.31) \text {, no } \mathrm{RT}>\text { after } \mathrm{BR}(\mathrm{OR}= \\
\text { 7.19), NS }\end{array}$ \\
\hline & & & & $\begin{array}{l}\text {-For physical well-being: no RT > prior to RT } \\
(\mathrm{OR}=4.43), \text { no } \mathrm{RT}>\text { after } \mathrm{BR}(\mathrm{OR}=3.97) \text {, } \\
\text { prior to } \mathrm{BR}<\text { after BR }(\mathrm{OR}=2.14) \text {, NS } \\
\text { - } \uparrow \mathrm{BMI} \downarrow \text { psychosocial and sexual well-being } \\
\text {-Younger patients }(<66 \mathrm{y}) \downarrow \text { psychosocial well- } \\
\text { being }\end{array}$ \\
\hline & $\begin{array}{l}\text { Jeevan et al, } \\
2017\end{array}$ & BREAST-Q & $\begin{array}{l}\text { Timing of reconstruction (IR vs DL); type of } \\
\text { reconstruction (implant vs pedicle tram vs }\end{array}$ & $\begin{array}{l}\text {-Pedicle TRAM } \uparrow \text { psychosocial, physical and } \\
\text { sexual well-being }\end{array}$ \\
\hline & & & DIEP FLAP vs free TRAM FLAP) & $\begin{array}{c}\text {-DIEP FLAP } \uparrow \text { psychosocial and physical well- } \\
\text { being; DL: free TRAM } \uparrow \text { sexual well-being }\end{array}$ \\
\hline & $\begin{array}{l}\text { Macadam et al, } \\
2010\end{array}$ & $\begin{array}{l}\text { QLQ-C30; BR23; } \\
\text { BREAST-Q }\end{array}$ & Type of implant (silicone vs saline) & -Silicone $\uparrow$ physical function $(O R=2.03)$ \\
\hline & $\begin{array}{l}\text { Potter et al, } \\
2009\end{array}$ & $\begin{array}{l}\text { QLQ-C30; BR23; } \\
\text { FACT-B }\end{array}$ & Complications & -NS \\
\hline & $\begin{array}{l}\text { Pusic et al, } \\
2017\end{array}$ & $\begin{array}{l}\text { BREAST-Q; } \\
\text { PROMIS-29 }\end{array}$ & $\begin{array}{l}\text { Type of reconstruction (implant vs FLAP); } \\
\text { implant procedure; type of autologous } \\
\text { reconstruction }\end{array}$ & $\begin{array}{l}\text {-FLAP } \uparrow \text { psychosocial and sexual well-being } \\
\text { but } \uparrow \text { pain interference }\end{array}$ \\
\hline & $\begin{array}{l}\text { Tonseth et al, } \\
2008\end{array}$ & SF-36 & Type of reconstruction (DIEP vs implant) & -NS \\
\hline & $\begin{array}{l}\text { Winters et al, } \\
2016\end{array}$ & $\begin{array}{l}\text { QLQ-C30; BR23; } \\
\text { FACT-B }\end{array}$ & $\begin{array}{l}\text { Type of reconstruction (implant vs FLAP); lymph } \\
\text { node positivity; BMI; type of axillary surgery; }\end{array}$ & $\begin{array}{l}-F L A P \uparrow \text { pain and } \uparrow \text { sexual function } \\
-R T \downarrow \text { social function }\end{array}$ \\
\hline & & & $\begin{array}{l}\text { tumor size; margin positivity; RT; CT; } \\
\text { complications; age }\end{array}$ & $\begin{array}{l}\text {-CT } \downarrow \text { global QoL, social functioning, } \\
\text { functional well-being, arm symptoms }\end{array}$ \\
\hline & & & & $\begin{array}{l}\text {-Early complications } \downarrow \text { global QoL, role } \\
\text { functioning, social functioning, physical } \\
\text { well-being, and functional well-being }\end{array}$ \\
\hline & & & & $\cdot \downarrow$ Age $\downarrow$ physical well-being \\
\hline & $\begin{array}{l}\text { Zhong et al, } \\
2011\end{array}$ & BREAST-Q & Timing of reconstruction (IR vs DL) & -NS \\
\hline & $\begin{array}{l}\text { Zhong et al, } \\
2016\end{array}$ & SF-36 & Timing of reconstruction (IR vs DL) & $\begin{array}{l}\text {-IR } \uparrow \text { psychosocial and sexual well-being, NS } \\
\text {-DL } \uparrow \text { physical well-being (chest), NS }\end{array}$ \\
\hline
\end{tabular}


TABLE 2 (Continued)

\begin{tabular}{|c|c|c|c|c|}
\hline $\begin{array}{l}\text { Critical } \\
\text { Appraisal }\end{array}$ & References & QoL Tools & Factors Affecting QoL & Results \\
\hline \multirow[t]{14}{*}{ Fair $(N=16)$} & $\begin{array}{l}\text { Bellino et al, } \\
2011\end{array}$ & SF-36 & $\begin{array}{l}\text { Depression; anxiety; work status; personality; } \\
\text { interpersonal problems; illness severity; illness } \\
\text { duration; number of childbirths; age at } \\
\text { diagnosis; age at reconstruction }\end{array}$ & $\begin{array}{l}\text {-Unemployed or housewife } \downarrow \text { QoL } \\
\text {-Harm avoidance } \uparrow \text { QoL (OR = 4.43) } \\
\text {-Vindictive/self-centered problems } \uparrow \text { QoL (OR } \\
\quad=2.14 \text { ) } \\
\text { - } \uparrow \text { Age at reconstruction } \uparrow \text { QoL }\end{array}$ \\
\hline & $\begin{array}{l}\text { Brandberg } \\
\text { et al, } 2000\end{array}$ & SF-36 & Type of reconstruction (TRAM vs LD vs LTD); RT & -NS \\
\hline & $\begin{array}{l}\text { Browne et al, } \\
2017\end{array}$ & $\begin{array}{l}\text { Constructed } \\
\text { questionnaire }\end{array}$ & $\begin{array}{l}\text { Complications; timing of reconstruction (IR vs } \\
\text { DL); type of reconstruction (implant vs FLAP) }\end{array}$ & $\begin{array}{l}\text {-FLAP with complications } \downarrow \text { emotional well- } \\
\text { being (OR }=3.56 \text { ), NS } \\
\text {-Implant-related complications } \downarrow \text { emotional } \\
\text { well-being (OR }=3.08), N S \\
\text {-Implant-related complications } \downarrow \text { physical } \\
\text { well-being (OR }=2.62) \text {, NS }\end{array}$ \\
\hline & $\begin{array}{c}\text { Cereijo-Garea } \\
\text { et al, } 2018\end{array}$ & BREAST-Q & $\begin{array}{l}\text { Age at interview; marital status; comorbidities; } \\
\text { anxiolytics; type of MT (NSSM VS SSM VS } \\
\text { MRM); simmetrization; lymphedema; RT; } \\
\text { immunotherapy }\end{array}$ & $\begin{array}{l}\text {-Comorbidities } \downarrow \text { psychosocial well-being } \\
\text { (OR }=2.10 \text { ), NS } \\
\text {-Divorced } \uparrow \text { psychosocial well-being (OR = } \\
\text { 6.90), NS } \\
\text {-Taking anxiolytic } \uparrow \text { sexual well-being (OR = } \\
\text { 3.48), NS } \\
\text {-SSM } \uparrow \text { physical well-being (chest) (OR = 4.27) } \\
\text {-Simmetrization } \uparrow \text { physical well-being (OR = } \\
\text { 2.49), NS } \\
\text {-Lymphedema } \downarrow \text { physical well-being (chest) } \\
\text { (OR = 12.9) } \\
\text { Immunotherapy } \downarrow \text { psychosocial (OR = 4.25) } \\
\text { and sexual well-being (OR = 7.34) }\end{array}$ \\
\hline & $\begin{array}{l}\text { Dean et al, } \\
2016\end{array}$ & BREAST-Q & $\begin{array}{l}\text { Timing of reconstruction (IR vs DL); type of } \\
\text { reconstruction (implant vs FLAP) }\end{array}$ & $\begin{array}{l}\text {-DL reconstruction } \uparrow \text { physical function } \\
\text { (chest), psychosocial and sexual well-being } \\
\text {-FLAP } \uparrow \text { psychosocial well-being }\end{array}$ \\
\hline & $\begin{array}{l}\text { Dieterich et al, } \\
2015\end{array}$ & BREAST-Q & $\begin{array}{l}\text { Type of implant (implant vs TILOOP); timing of } \\
\text { reconstruction (IR vs DL); type of MT (SSM or } \\
\text { NSSM vs MRM vs SMT); additional FLAP; age } \\
\text { at reconstruction; previous breast surgery; } \\
\text { complications; seroma; lymph node dissection; } \\
\text { CT; RT; BMI; smoking status }\end{array}$ & $\begin{array}{l}\text {-TILOOP } \uparrow \text { physical well-being (chest) (OR = } \\
\text { 5.31), NS } \\
\text {-Previous breast surgery } \uparrow \text { physical and } \\
\text { psychosocial well-being } \\
\text { - } \uparrow \text { BMI } \downarrow \text { physical well-being } \\
\text {-Smoking } \downarrow \text { physical well-being }\end{array}$ \\
\hline & Juhl et al, 2017 & SWLS & $\begin{array}{l}\text { Personality; prereconstruction QoL; age at } \\
\text { interview; BMI; marital status; educational } \\
\text { level; laterality of reconstruction; } \\
\text { complications (minor vs major); RT; CT; type of } \\
\text { reconstruction (implant vs FLAP); timing of } \\
\text { reconstruction (IR vs DL) }\end{array}$ & $\begin{array}{l}-\uparrow \text { Neuroticism } \downarrow \text { QoL } \\
-\uparrow \text { Openness } \downarrow \text { QoL } \\
-\uparrow \text { prereconstruction QoL } \uparrow \text { QoL after BR } \\
-\uparrow \mathrm{BMI} \downarrow \text { QoL }\end{array}$ \\
\hline & Liu et al, 2014 & BREAST-Q & $\begin{array}{l}\text { Complications; type of reconstruction (implant vs } \\
\text { FLAP) }\end{array}$ & $\begin{array}{l}\text {-Complication } \downarrow \text { physical well-being } \\
\text {-Flap } \uparrow \text { psychosocial }(\mathrm{OR}=3.02) \text { and sexual } \\
\text { well-being }(\mathrm{OR}=3.25)\end{array}$ \\
\hline & Oh et al, 2018 & FACT-B & $\begin{array}{l}\text { Timing of reconstruction (IR vs DL); type of } \\
\text { reconstruction (implant vs FLAP) }\end{array}$ & -NS \\
\hline & $\begin{array}{l}\text { Parra Pont } \\
\text { et al, } 2017\end{array}$ & BREAST-Q & RT & -RT $\downarrow$ physical well-being (chest) \\
\hline & $\begin{array}{l}\text { Peiris et al, } \\
2017\end{array}$ & BREAST-Q & Timing of RT (after BR; before BR) & -NS \\
\hline & $\begin{array}{l}\text { Pinell-White } \\
\text { et al, } 2015\end{array}$ & WHO-QOL BREF & $\begin{array}{l}\text { Age at interview; race; educational level; marital } \\
\text { status; community setting; work status; } \\
\text { insurance type; cancer stage; complications; } \\
\text { obesity; RT; type of reconstruction (implant vs } \\
\text { LD vs TRAM); additional surgery }\end{array}$ & $\begin{array}{l}\text { - } \uparrow \text { Educational level } \uparrow \text { psychological losses } \\
\text {-Married } \downarrow \text { overall QoL, social and } \\
\text { environmental domains } \\
\text {-Advanced stages } \downarrow \text { satisfaction with life }\end{array}$ \\
\hline & $\begin{array}{l}\text { Pirro et al, } \\
2017\end{array}$ & BREAST-Q & Type of reconstruction (implant vs FLAP) & $\begin{array}{l}\text {-Implant } \uparrow \text { chest physical well-being (OR = } \\
\text { 2.81), NS }\end{array}$ \\
\hline & $\begin{array}{l}\text { Qureshi et al, } \\
2017\end{array}$ & BREAST-Q & Implant procedure; fat grafting & -NS \\
\hline
\end{tabular}


TABLE 2

(Continued)

\begin{tabular}{|c|c|c|c|}
\hline \multirow[t]{2}{*}{$\begin{array}{l}\text { Critical } \\
\text { Appraisal }\end{array}$} & References & QoL Tools & Factors Affecting QoL \\
\hline & $\begin{array}{l}\text { Santosa et al, } \\
2018\end{array}$ & BREAST-Q & $\begin{array}{l}\text { Type of reconstruction (implant vs FLAP); age; } \\
\text { BMI; prereconstruction QoL; time after } \\
\text { reconstruction; laterality of reconstruction; } \\
\text { lymph node dissection; type of MT (NSSM vs } \\
\text { MRM); diabetes; smoking status; RT; CT; race, } \\
\text { ethnicity; educational level; level of income; } \\
\text { marital status; work status }\end{array}$ \\
\hline & $\begin{array}{l}\text { Sisco et al, } \\
2015\end{array}$ & BREAST-Q & Age at interview $(<65>)$ \\
\hline \multirow[t]{7}{*}{ Poor $(N=9)$} & $\begin{array}{l}\text { Ashraf et al, } \\
2013\end{array}$ & $\mathrm{SF}-12$ & Paternalistic, informed, or shared decision \\
\hline & $\begin{array}{l}\text { Bailey et al, } \\
2017\end{array}$ & BREAST-Q & $\begin{array}{l}\text { Type of MT; quality of care; complications; } \\
\text { prereconstruction QoL }\end{array}$ \\
\hline & $\begin{array}{l}\text { De Gournay } \\
\text { et al } 2010\end{array}$ & QLQ-C30; BR23 & $\begin{array}{l}\text { Type of reconstruction (implant vs FLAP); } \\
\text { complications }\end{array}$ \\
\hline & $\begin{array}{l}\text { Heneghan } \\
\text { et al, } 2011\end{array}$ & $\begin{array}{l}\text { QLQ-C30; BR23; } \\
\text { FACT-B }\end{array}$ & $\begin{array}{l}\text { RT; type of reconstruction (LD alone and LD with } \\
\text { implant); cancer stage }\end{array}$ \\
\hline & $\begin{array}{l}\text { Ludolph et al, } \\
2015\end{array}$ & BREAST-Q & Age at interview $(<60<)$ \\
\hline & $\begin{array}{l}\text { Matthews } \\
\text { et al, } 2017\end{array}$ & QLQ-C30 & $\begin{array}{l}\text { Age at interview; type of reconstruction (implant } \\
\text { vs FLAP); time after reconstruction; } \\
\text { perceptions on scaring; satisfaction with } \\
\text { outcome; psychosocial and sexual well-being }\end{array}$ \\
\hline & $\begin{array}{l}\text { Menez et al, } \\
2017\end{array}$ & BREAST-Q & $\begin{array}{l}\text { Timing of reconstruction (IR vs DL); DL } \\
\text { reconstruction (failure vs conversion); type of } \\
\text { reconstruction (DIEP, LDI, LD); complications } \\
\text { (minor vs major); age }\end{array}$ \\
\hline
\end{tabular}

Critical

Appraisal

Sisco et

Ashraf
2013

Bailey et al,

De Gournay

2015

Matthews

Menez et al,

Very Poor ( $\mathrm{N}$

$=3$ )
Thorarinsson

et al, 2017

SF-36; PGWB;

EQ-5D;

BREAST-Q

Yang et al, SF-36

2015
QLQ-C30;

SCL-R-90; SF-36
Type of reconstruction (DIEP vs LD vs LDT vs implant)

RT; lymph node dissection

Timing of reconstruction (IR vs DL)

Min et al, $2010 \quad$ BR-23

$\begin{array}{ll}\text { oktas et al, } & \text { QLQ-C30; } \\ 2011 & \text { SCL-R-90; SF-36 }\end{array}$

Results

-FLAP $\uparrow$ higher psychosocial and sexual wellbeing

- $\uparrow$ Age $\uparrow$ psychosocial and sexual well-being

- $\uparrow \mathrm{BMI} \downarrow$ physical well-being

- $\uparrow$ Prereconstruction QoL $\uparrow$ psychosocial, physical and sexual well-being after reconstruction

- $\uparrow$ Time after reconstruction $\uparrow$ psychosocial, physical and sexual well-being

-NSSM $\uparrow$ psychosocial and sexual well-being

-Ex-smokers $\downarrow$ psychosocial and sexual wellbeing

-RT after reconstruction $\downarrow$ psychosocial, physical and sexual well-being

-Chemotherapy $\downarrow$ psychosocial and sexual well-being

-Black race $\uparrow$ psychosocial and sexual wellbeing

-College $\downarrow$ sexual well-being

-Married $\uparrow$ psychosocial and sexual well-being

-Part-time $\uparrow$ physical well-being

-NS $(\rho=0.12)$

-Paternalistic $\downarrow$ physical function (OR $=4.12)$

-NSPM $\uparrow$ psychosocial well-being

$$
(\mathrm{OR}=2.57)
$$

-NS

-LD alone $\uparrow$ QLQC-30 global score (OR = 12.90), NS

-NS

- $\uparrow$ Perceptions on scar quality $\uparrow$ overall QoL

- $\uparrow$ Satisfaction $\uparrow$ overall QoL

- $\uparrow$ Psychosocial well-being $\uparrow$ overall QoL

- $\uparrow$ Sexual well-being $\uparrow$ overall QoL

-IR $\uparrow$ sexual well-being (OR = 129.2)

-Failure reconstruction $\uparrow$ sexual well-being (OR = 5.50), NS

-LDI $\uparrow$ sexual well-being (OR = 4.12), NS

-Major complications $\downarrow$ psychosocial and sexual well-being

- Older patients (>60) $\uparrow$ psychosocial wellbeing

-DIEP $\downarrow$ vitality

$-N S$

-DL $\downarrow$ physical (OR = 5.12), emotional function (OR = 2.62) and $\uparrow$ body pain (OR $=2.62$ )

Cancer stage; age $(<40<)$; postop RT; postop CT; Neo-CT
-Lower stages $\downarrow$ body image (OR = 15.20), sexual functioning (OR = 52.15); and future perspectives (OR $=4.76$ ), NS

-No RT $\uparrow$ sexual functioning (OR = 3.62), and future perspectives (OR $=2.26$ ), NS -Neo-CT $\downarrow$ future perspectives (OR $=5.50)$ 
TABLE 2 (Continued)

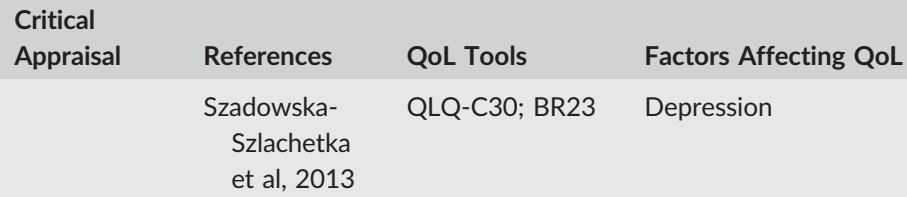

References

Szadowska-

Szlachetka

et al, 2013

QoL Tools

QLQ-C30; BR23

Factors Affecting QoL

Depression

Results

-Depression $\downarrow$ overall QoL (OR $=10.38)$, physical $(O R=5.31)$, role $(O R=4.67)$, emotional $(O R=9.14)$, and cognitive function $(O R=8.66)$

Abbreviations: BMI, Body mass index; BREAST-Questionnaire; DIEP, deep inferior epigastric perforators flap; DL, delayed reconstruction; EORTC QLQC30, The European Organization for Research and Treatment of Cancer (General module); EQ-5D, The EuroQol Group questionnaire; FACT-B, The Functional Assessment of Cancer Therapy-Breast; IR, immediate reconstruction; LD, latissimus dorsi myocutaneous flap; LDI, latissimus dorsi myocutaneous flap with implant; LTD, lateral thoracodorsal flap; MRM, modified radical mastectomy; neo-CT, neoadjuvant chemotherapy; NS, not statistically significant ( $P$ > .05); NSPM, nipple-sparing mastectomy; NSSM, nonskin sparing mastectomy; OR, odds ratio; PGWB, Psychological General Well-Being Index; postop CT, postoperative chemotherapy; postop RT, postoperative radiotherapy; QLQ-BR23, Supplementary questionnaire (breast cancer module); SF-36, The 36Item Short Form Health Survey questionnaire; SF-12, an abridged version of the SF-36; Satisfaction with Life Scale (SWLS); SMT, subcutaneous mastectomy; SSM, skin-sparing mastectomy; TILOOP, titanium-coated polypropylene mesh implant; TRAM, transverse rectus abdominis myocutaneous flap; WHO-QOL-BREF, World Health Organization Quality of Life questionnaire.

Four studies out of six (67\%) reported that chemotherapy was associated with low levels of psychosocial, sexual, and social function, and an overall lower quality of life..$^{6,37,52,53}$ One study found that patients who underwent immunotherapy presented higher odds of having lower psychosocial and sexual well-being. ${ }^{10}$

Two studies out of four (50\%) found that less invasive stages were associated with higher levels of overall quality and satisfaction with life.,31 However, further analysis revealed that patients with advanced stages of cancer had higher odds of worse overall quality of life, especially for sexual function. ${ }^{53}$

Presence of lymphedema was identified as a significant predictor of lower physical well-being on the chest, ${ }^{10}$ role function, cognitive function, and body image. ${ }^{2}$

Five studies out of six (83\%) reported that a higher BMI significantly affected the overall quality of life and its physical, psychosocial and sexual dimensions. ${ }^{31,37,39,52}$ Regarding smoking status, both studies that identified this variable consistently reported its negative effects on physical, psychosocial, and sexual function. ${ }^{37,39}$

Lastly, one study ${ }^{10}$ also found that patients who took anxiolytics also presented higher odds of having lower sexual well-being after breast reconstruction.

\section{9 | Psychosocial variables}

Prereconstruction quality of life, psychosocial and sexual well-being, depression, personality, interpersonal relationships, satisfaction with the cosmetic outcome, perceptions on scaring, and the process of decision-making were identified across the included studies as factors affecting quality of life.

Two studies out of three (67\%) found that higher levels of prereconstruction quality of life were significant predictors of higher levels of overall quality of life ${ }^{36}$ and its dimensions such as psychosocial, physical, and sexual well-being. ${ }^{32,37}$

The association between depression symptoms and quality of life was identified by two studies. One study did not find depression to be a significant predictor of overall quality of life, ${ }^{27}$ while the other study found that higher depression levels were significantly associated with lower overall quality of life, physical, role, emotional, and cognitive function, with large OR. ${ }^{54}$

Two studies focused on the role of personality characteristics on self-reported quality of life. Results revealed that higher levels of harm avoidance were associated with higher levels of overall quality of life ${ }^{27}$ while high neuroticism and openness were associated with lower overall quality of life. ${ }^{32}$

Satisfaction with esthetic outcome and scar quality were also associated with higher levels of overall quality of life. ${ }^{31,50}$

Lastly, one study found that patients with a paternalistic decisionmaking process (vs informed) reported lower levels of physical function. ${ }^{55}$

\subsection{0 | Patients sociodemographic characteristics}

Age, marital status, educational level, work status, and race were the sociodemographic characteristics that presented significant results related to quality of life after breast reconstruction.

Six studies out of 14 (43\%) identified age as a significant factor. Three studies found that older patients reported higher levels of psychosocial, sexual, and physical well-being, ${ }^{6,37,41}$ while Dauplat et al $^{31}$ found that younger patients reported higher overall quality of life, but with low effect sizes. Two studies suggested that older patients reported higher levels of overall quality of life after breast reconstruction. ${ }^{27,39}$ The remaining studies did not report significant differences between younger and older patients. 3,10,32,50,52,53,56,57

Four studies reported findings regarding marital status. One study reported that married women reported lower levels of overall quality of life, ${ }^{3}$ while another study reported somewhat opposite results, since married women reported higher levels of psychosocial and sexual well-being. ${ }^{37}$ Juhl and colleagues ${ }^{32}$ did not find any differences regarding marital status, but Cereijo-Garea and colleagues ${ }^{10}$ found that divorced women had higher odds of having higher psychosocial well-being, when compared with single women. 
Two studies out of three (67\%) suggested that women with higher levels of education reported lower levels of psychological and sexual function. $^{3,37}$

Work status was studied by four studies. Three of them (75\%) found that active women reported higher levels of quality of life, ${ }^{27,31}$ especially if working in part-time jobs. ${ }^{37}$

Lastly, two studies reported results regarding race, ${ }^{3,37}$ and one of them reported significant differences favoring black women for psychosocial and sexual well-being. ${ }^{37}$

\section{4 | DISCUSSION}

Since the percentage of patients who underwent breast reconstruction increased from $26.9 \%$ in 2005 to $43 \%$ in $2014,{ }^{58}$ the assessment of these patient's point of view is considered a further step towards a more integrated approach of cancer treatment.

To the best of our knowledge, the present study is the first systematic review of the literature that explores the relationship between surgical, clinical, psychosocial, and sociodemographic variables and self-reported quality of life after postmastectomy breast reconstruction in breast cancer patients.

Which factors affect quality of life after breast reconstruction?

This review identified 32 empirically based variables associated with self-reported domains of quality of life in women with breast cancer after reconstruction.

Some variables were more prominent than others as they presented a higher number of appearances across studies, such as type of reconstruction, time of reconstruction, surgical-related complications, and postreconstruction radiotherapy. This finding sustains the argument that research focused on quality of life after breast reconstruction is still focused on surgical variables and their impact on the patients' quality of life outcomes. However, interesting results were found for psychosocial, clinical, and sociodemographic variables and, thus, also worthy of discussion.

This review found that decision-making process may affect patients' self-reported quality of life after breast reconstruction. Patients within the paternalistic model, which represents a physician dominated communication, reported lower physical function compared with the patients who took a more active role in their treatment choices. This result sustains the need to increase the patients' information about this procedure in order to prevent decisional-regret, a common phenomenon after breast reconstruction. ${ }^{59}$

Personality traits also had significant effects on the patient's overall quality of life after breast reconstruction, specially neuroticism. ${ }^{32}$ This personality trait has previously been reported as a risk factor for overall quality of life in the general population. ${ }^{60}$ This finding suggests that these characteristics should be carefully evaluated before surgery so that precise referrals can be made to psycho-oncology units to help these patients develop better coping mechanisms. The same principle should be applied when depressive symptoms are present before reconstruction, since this review also suggested that this variable is negatively associated with physical, role, emotional, and cognitive function after this procedure. ${ }^{54}$

This review also suggested that prereconstruction levels of psychosocial and sexual functioning may also help predict postreconstruction quality of life. ${ }^{32,36,37}$ Hereafter, prospective studies are also needed to explore which psychosocial, clinical, demographic, and surgical variables may influence these patients' postreconstruction quality of life.

In addition, current results have congruently proposed higher BMI as a possible risk factor for lower quality of life. ${ }^{31,32,39}$ A recent metaanalysis also supported the suggested impact of obesity on morbidity and surgical complications after reconstruction. ${ }^{61}$

When considering sociodemographic variables, age is an important variable to discuss. A recent systematic review has reported that the uptake of breast reconstruction after mastectomy is lower in older patients, raising the question that some older women are currently not proposed for reconstruction because of generalized assumptions about esthetic needs or age-related safety concerns. ${ }^{62}$ Interestingly, this review suggested that patients with 60 years old or more reported higher levels of psychosocial well-being after this surgery, ${ }^{22,34}$ or similar overall quality of life results when compared with younger patients. ${ }^{55,58}$ Similar results have been previously reported. ${ }^{63}$ Therefore, this review seems to suggest that age alone is not a contraindication for quality of life after breast reconstruction. Instead, it supports the idea that older women can benefit from reconstruction, and this procedure should be offered and openly discussed with them.

Previous research also suggests that married women are more likely to undergo breast reconstruction than single women. ${ }^{64}$ Future research should focus on women's relationship motivations for breast reconstruction and on the expected impact of this surgery on the couple's attachment and marital adjustment.

\section{I CONCLUSION}

The results' section of this review identified a broad collection of 32 empirically-based factors affecting quality of life grouped into four different categories: surgical, clinical, psychosocial, and sociodemographic variables. Therefore, it sustains the need to adopt a more holistic approach and advises the consideration of preoperative psychosocial, clinical, and sociodemographic variables to better understand these patient's overall quality of life and to implement future preventive measures. Its new contribution is that it goes beyond surgical factors of morbidity and uses patient reported outcomes to scope wider issues that influence quality of life, particularly preoperative psychosocial, clinical, and sociodemographic factors.

\section{1 | Study limitations}

The consideration of PROMs for the assessment of quality of life resulted on the exclusion of qualitative studies, and, as a result, relevant content may have been excluded. Also, some of the included studies presented associations with global quality of life measures. 
Since quality of life is a broad concept, an effort was made to discriminate between its different dimensions throughout this review, although this was not always possible. Most of the included studies used crosssectional retrospective designs, but this methodology is susceptible to a high error rate and bias. Most papers did not control for confounding factors on their regression models. Studies with poor and very poor quality were included in this review, but the evaluation of quality was not considered when reporting the results, which may increase the risk of bias. Therefore, results should be carefully interpreted, and further prospective, higher quality, larger studies, with instruments discriminating the different quality of life domains, such as the BREAST-Q breast reconstruction module, should be utilized.

\section{2 | Clinical implications}

Findings regarding modifiable factors such as psychosocial and clinical variables suggested that patients who are proposed to breast reconstruction may benefit from a preoperative assessment in order to identify possible vulnerability factors for worse quality of life. Preoperative psychosocial assessment is already implemented as a routine practice in other clinical settings such as bariatric surgery, ${ }^{65}$ organ transplantation, ${ }^{66}$ and other cosmetic surgeries. ${ }^{67}$ These assessments can help identify patients at risk for lower quality of life, which patients are more likely to benefit from this procedure, and help the identification of areas for psychosocial intervention, both before and after reconstruction. This may also include the need to discuss breast reconstruction outcomes, including the negative ones, in order to give these women more realistic expectations, as suggested in a previous review. ${ }^{22}$

Other clinical modifiable factors, such as weight and smoking status, should also be carefully addressed preoperatively, reinforcing the possible benefits of adopting preventive measures. Additional research is needed to determine if weight and tobacco reductions prior to reconstruction improve postoperative quality of life and if endocrine therapy poses and additional risk to quality of life after this procedure.

\section{ACKNOWLEDGEMENTS}

This research did not receive any specific grant from funding agencies in the public, commercial, or not-for-profit sectors.

\section{DISCLOSURE STATEMENT}

No potential conflict of interest to declare.

\section{ORCID}

M. Graça Pereira (1) https://orcid.org/0000-0001-7987-2562

\section{REFERENCES}

1. Schmauss D, Machens HG, Harder Y. Breast reconstruction after mastectomy. Front Surg. 2016;2:71. https://doi.org/10.3389/ fsurg.2015.00071

2. Penha TR, Botter B, Heuts EM, Voogd AC, von Meyenfeldt MF, van der Hulst RR. Quality of life in patients with breast cancer-related lymphedema and reconstructive breast surgery. J Reconstr Microsurg. 2016;32(6):484-490. https://doi.org/10.1055/s-0036-1572538

3. Pinell-White XA, Duggal C, Metcalfe D, Sackeyfio R, Hart AM, Losken A. Patient-reported quality of life after breast reconstruction: a one-year longitudinal study using the WHO-QOL survey. Ann Plast Surg. 2015;75(2):144-148. https://doi.org/10.1097/SAP.00000000 00000065

4. Nissen MJ, Swenson KK, Ritz LJ, Farrell JB, Sladek ML, Lally RM. Quality of life after breast carcinoma surgery: A comparison of three surgical procedures. Cancer. 2001;91(7):1238-1246.

5. Pusic AL, Matros E, Fine N, et al. Patient-reported outcomes 1 year after immediate breast reconstruction: results of the mastectomy reconstruction outcomes consortium study. J Clin Oncol. 2017;35(22):2499-2506. https://doi.org/10.1200/JCO.2016.69.9561

6. Winters ZE, Afzal M, Balta V, et al. Patient-reported outcomes and their predictors at 2- and 3-year follow-up after immediate latissimus dorsi breast reconstruction and adjuvant treatment. $\mathrm{Br} J$ Surg. 2016;103(5):524-536. https://doi.org/10.1002/bjs.10102

7. Heimes AS, Stewen K, Hasenburg A. Psychosocial aspects of immediate versus delayed breast reconstruction. Breast Care (Basel). 2017;12(6):374-377. https://doi.org/10.1159/000485234

8. Lee C, Sunu C, Pignone M. Patient-reported outcomes of breast reconstruction after mastectomy: a systematic review. J Am Coll Surg. 2009;209(1):123-133. https://doi.org/10.1016/j.jamcollsurg.2009. 02.061

9. Parker PA, Baile WF, de Moor C, Cohen L. Psychosocial and demographic predictors of quality of life in a large sample of cancer patients. Psychooncology. 2003;12(2):183-193.

10. Cereijo-Garea C, Pita-Fernández S, Acea-Nebril B, et al. Predictive factors of satisfaction and quality of life after immediate breast reconstruction using the BREAST-Q. J Clin Nurs. 2018;27(78):1464-1474. https://doi.org/10.1111/jocn.14291

11. Platt J, Zhong T. Patient-centered breast reconstruction based on health-related quality-of-life evidence. Clin Plast Surg. 2018;45(1):137-143. https://doi.org/10.1016/j.cps.2017.08.011

12. Winters ZE, Benson JR, Pusic AL. A systematic review of the clinical evidence to guide treatment recommendations in breast reconstruction based on patient- reported outcome measures and healthrelated quality of life. Ann Surg. 2010;252(6):929-942. https://doi. org/10.1097/SLA.0b013e3181e623db

13. Menezes MM, Bello MA, Millen E, et al. Breast reconstruction and risk of lymphedema after mastectomy: a prospective cohort study with 10 years of follow-up. J Plast Reconstr Aesthet Surg. 2016; 69(9):1218-1226. https://doi.org/10.1016/j.bjps.2016.06.001

14. Zhong T, Hu J, Bagher S, et al. A comparison of psychological response, body image, sexuality, and quality of life between immediate and delayed autologous tissue breast reconstruction: a prospective longterm outcome study. Plast Reconstr Surg. 2016;138(4):772-780. https://doi.org/10.1097/PRS.0000000000002536

15. Liu C, Zhuang Y, Momeni A, et al. Quality of life and patient satisfaction after microsurgical abdominal flap versus staged expander/ implant breast reconstruction: a critical study of unilateral immediate breast reconstruction using patient-reported outcomes instrument BREAST-Q. Breast Cancer Res Treat. 2014;146(1):117-126. https:// doi.org/10.1007/s10549-014-2981-z

16. Billig J, Jagsi R, Qi J, et al. Should immediate autologous breast reconstruction be considered in women who require post-mastectomy radiation therapy? A prospective analysis of outcomes. Plast Reconstr Surg. 2017;139(6):1279-1288. https://doi.org/10.1097/PRS. 0000000000003331 
17. Jagsi R, Momoh AO, Qi J, et al. Impact of radiotherapy on complications and patient-reported outcomes after breast reconstruction. J Natl Cancer Inst. 2018;110(2):157-165. https://doi.org/10.1093/jnci/ djx148

18. Kanatas A, Velikova G, Roe B, et al. Patient-reported outcomes in breast oncology: a review of validated outcome instruments. Tumori. 2012;98(6):678-688. https://doi.org/10.1700/1217.13489

19. Donovan K, Sanson-Fisher RW, Redman S. Measuring quality of life in cancer patients. J Clin Oncol. 1989;7(7):959-968.

20. WHOQOL Measuring Quality Of Life. The World Health Organization Quality Of Life. 1997. https://www.who.int/mental_health/ media/68.pdf.

21. Nahabedian MY. Shaped versus round implants for breast reconstruction: indications and outcomes. Plast Reconstr Surg Glob Open. 2014;2(3):e116. https://doi.org/10.1097/GOX.0000000000000068

22. Pusic AL, Klassen AF, Snell L, et al. Measuring and managing patient expectations for breast reconstruction: impact on quality of life and patient satisfaction. Expert Rev Pharmacoecon Outcomes Res. 2012;12(2):149-158. https://doi.org/10.1586/erp.11.105

23. Fingeret MC, Nipomnick SW, Crosby MA, Reece GP. Developing a theoretical framework to illustrate associations among patient satisfaction, body image and quality of life for women undergoing breast reconstruction. Cancer Treat Rev. 2013;39(6):673-681. https:// doi.org/10.1016/j.ctrv.2012.12.010

24. Liberati A, Altman DG, Tetzlaff J, et al. The PRISMA statement for reporting systematic reviews and meta-analyses of studies that evaluate healthcare interventions: explanation and elaboration. BMJ. 2009;339(jul21 1):b2700. https://doi.org/10.1136/bmj.b2700

25. Moher D, Liberati A, Tetzlaff J, Altman DG, PRISMA Group. Preferred reporting items for systematic reviews and meta-analyses: the PRISMA statement. PLoS Med. 2009;6(7):e1000097. https://doi.org/10.1371/ journal.pmed.1000097

26. Oh DD, Flitcroft K, Brennan ME, Snook KL, Spillane AJ. Patientreported outcomes of breast reconstruction in older women: audit of a large metropolitan public/private practice in Sydney, Australia. Psychooncology. 2018;27(12):2815-2822. https://doi.org/10.1002/ pon.4895

27. Bellino S, Fenocchio M, Zizza M, Rocca G, Bogetti P, Bogetto F. Quality of life of patients who undergo breast reconstruction after mastectomy: effects of personality characteristics. Plast Reconstr Surg. 2011;127(1):10-17. https://doi.org/10.1097/PRS.0b013e3181f956c0

28. Brandberg $\mathrm{Y}$, Malm M, Blomqvist L. A prospective and randomized study, "SVEA," comparing effects of three methods for delayed breast reconstruction on quality of life, patient-defined problem areas of life, and cosmetic result. Plast Reconstr Surg. 2000;105(1):66-74.

29. Bennett KG, Qi J, Kim HM, et al. Association of fat grafting with patient-reported outcomes in postmastectomy breast reconstruction. JAMA Surg. 2017;152(10):944-950. https://doi.org/10.1001/ jamasurg.2017.1716

30. Dean NR, Crittenden T. A five-year experience of measuring clinical effectiveness in a breast reconstruction service using the BREAST-Q patient reported outcomes measure: a cohort study. JPRAS: Int J Sur Rec. 2016;69(11):1469-1477. https://doi.org/10.1016/j.bjps.2016. 08.015

31. Dauplat J, Kwiatkowski F, Rouanet P, et al. Quality of life after mastectomy with or without immediate breast reconstruction. $\mathrm{Br} J$ Surg. 2017;104(9):1197-1206. https://doi.org/10.1002/bjs.10537

32. Juhl AA, Damsgaard TE, O'Connor M, Christensen S, Zachariae R. Personality traits as predictors of quality of life and body image after breast reconstruction. Plast Reconstr Surg Glob Open. 2017;5(5): e1341. https://doi.org/10.1097/GOX.0000000000001341
33. Zhong T, McCarthy C, Min S, et al. Patient satisfaction and healthrelated quality of life after autologous tissue breast reconstruction: a prospective analysis of early postoperative outcomes. Cancer. 2012;118(6):1701-1709. https://doi.org/10.1002/cncr.26417

34. Yang JD, Huh JS, Min YS, Kim HJ, Park HY, Jung TD. Physical and functional ability recovery patterns and quality of life after immediate autologous latissimus dorsi breast reconstruction: a 1-year prospective observational study. Plast Reconstr Surg. 2015;136(6):1146-1154. https://doi.org/10.1097/PRS.0000000000001769

35. Sullivan GM, Feinn R. Using effect size-or why the $p$ value is not enough. J Grad Med Educ. 2012;4(3):279-282. https://doi.org/ 10.4300/JGME-D-12-00156.1

36. Bailey CR, Ogbuagu O, Baltodano PA, et al. Quality-of-life outcomes improve with nipple-sparing mastectomy and breast reconstruction. Plast Reconstr Surg. 2017;140(2):219-226. https://doi.org/10.1097/ PRS.0000000000003505

37. Santosa KB, Qi J, Kim HM, Hamill JB, Wilkins EG, Pusic AL. Long-term patient-reported outcomes in postmastectomy breast reconstruction. JAMA Surg. 2018;153(10):891-899. https://doi.org/10.1001/ jamasurg.2018.1677

38. Beugels J, Kool M, Hoekstra LT, et al. Quality of life of patients after immediate or delayed autologous breast reconstruction: a multicenter study. Ann Plast Surg. 2018;81(5):523-527. https://doi.org/10.1097/ SAP. 0000000000001618

39. Dieterich M, Angres J, Stachs A, et al. Patient-report satisfaction and health-related quality of life in TiLOOP $®$ Bra-assisted or implant-based breast reconstruction alone. Aesthetic Plast Surg. 2015;39(4):523-533. https://doi.org/10.1007/s00266-015-0520-x

40. Browne JP, Jeevan R, Gulliver-Clarke C, Pereira J, Caddy CM, van der Meulen JHP. The association between complications and quality of life after mastectomy and breast reconstruction for breast cancer. Cancer. 2017 Sep 15;123(18):3460-3467. https://doi.org/10.1002/ cncr.30788

41. Ménez T, Michot A, Tamburino S, Weigert R, Pinsolle V. Multicenter evaluation of quality of life and patient satisfaction after breast reconstruction, a long-term retrospective study. Ann Chir Plast Esthet. 2018;63(2):126-133. https://doi.org/10.1016/j.anplas.2017.07.022

42. Jeevan R, Browne JP, Gulliver-Clarke C, et al. Surgical determinants of patient-reported outcomes following postmastectomy reconstruction in women with breast cancer. Plast Reconstr Surg. 2017;139(5):1036e-1045e. https://doi.org/10.1097/PRS.00000000 00003236

43. Goktas SB, Gulluoglub BM, Selimen D. Immediate or delayed breast reconstruction after radical mastectomy in breast cancer patients: does it make a difference in the quality of life. Turkiye Klinikleri J Med Sci. 2011;31(3):664-673. https://doi.org/10.5336/medsci.2009-15488

44. Heneghan HM, Prichard RS, Lyons R, et al. Quality of life after immediate breast reconstruction and skin-sparing mastectomy-a comparison with patients undergoing breast conserving surgery. Eur J Surg Oncol. 2011;37(11):937-943. https://doi.org/10.1016/j.ejso. 2011.08.126

45. Macadam SA, Zhong T, Weichman K, et al. Quality of life and patientreported outcomes in breast cancer survivors: a multicenter comparison of four abdominally based autologous reconstruction methods. Plast Reconstr Surg. 2016;137(3):758-771. https://doi.org/10.1097/ 01.prs.0000479932.11170.8f

46. Thorarinsson A, Fröjd V, Kölby L, Lidén M, Elander A, Mark H. Patient determinants as independent risk factors for postoperative complications of breast reconstruction. Gland Surg. 2017;6(4):355-367. https://doi.org/10.21037/gs.2017.04.04 
47. Qureshi AA, Odom EB, Parikh RP, Myckatyn TM, Tenenbaum MM. Patient-Reported Outcomes of aesthetics and satisfaction in immediate breast reconstruction after nipple-sparing mastectomy with implants and fat grafting. Aesthet Surg J. 2017;37(9):999-1008. https://doi.org/10.1093/asj/sjx048

48. Macadam SA, Ho AL, Cook EF Jr, Lennox PA, Pusic AL. Patient satisfaction and health-related quality of life following breast reconstruction: patient-reported outcomes among saline and silicone implant recipients. Plast Reconstr Surg. 2010;125(3):761-771. https:// doi.org/10.1097/PRS.0b013e3181cb5cf8

49. Macadam SA, Ho AL, Lennox PA, Pusic AL. Patient-reported satisfaction and health-related quality of life following breast reconstruction: a comparison of shaped cohesive gel and round cohesive gel implant recipients. Plast Reconstr Surg. 2013;131(3):431-441. https://doi.org/ 10.1097/PRS.0b013e31827c6d55

50. Matthews H, Turner A, Williamson I, Clyne W. 'It's a silver lining': a template analysis of satisfaction and quality of life following post-mastectomy breast reconstruction. $\mathrm{Br} J$ Health Psychol. 2018;23(2):455-475. https://doi.org/10.1111/bjhp.12299

51. Pont LP, Marcelli S, Robustillo M, et al. immediate breast reconstruction with abdominal free flap and adjuvant radiotherapy: evaluation of quality of life and outcomes. Plast Reconstr Surg. 2017;140(4):681-690. https://doi.org/10.1097/PRS.0000000000003664

52. Eriksson M, Anveden L, Celebioglu F, et al. Radiotherapy in implantbased immediate breast reconstruction: risk factors, surgical outcomes, and patient-reported outcome measures in a large Swedish multicenter cohort. Breast Cancer Res Treat. 2013;142(3):591-601. https://doi.org/ 10.1007/s10549-013-2770-0

53. Min SY, Kim HY, Jung SY, et al. Oncological safety and quality of life associated with mastectomy and immediate breast reconstruction with a latissimus dorsi myocutaneous flap. Breast J. 2010;16(4):356-361. https://doi.org/10.1111/j.1524-4741.2010.00941.x

54. Szadowska-Szlachetka ZC, Stanislawek A, Kachaniuk H, et al. Occurrence of depression symptoms measured by the Beck Depression Inventory (BDI) in women after mastectomy and breast reconstruction with regard to the assessment of quality of life. Przeglad Menopauzalny. 2013;17(4):293-299. https://doi.org/10.5114/pm.2013.37843

55. Ashraf AA, Colakoglu S, Nguyen JT, et al. Patient involvement in the decision-making process improves satisfaction and quality of life in postmastectomy breast reconstruction. J Surg Res. 2013; 184(1):665-670. https://doi.org/10.1016/j.jss.2013.04.057

56. Ludolph I, Horch RE, Harlander M, et al. Is there a rationale for autologous breast reconstruction in older patients? A retrospective single center analysis of quality of life, complications and comorbidities after DIEP or ms-TRAM flap using the BREAST-Q. Breast J. 2015;21(6):588-595. https://doi.org/10.1111/tbj.12493

57. Sisco M, Johnson DB, Wang C, Rasinski K, Rundell VL, Yao KA. The quality-of-life benefits of breast reconstruction do not diminish with age. J Surg Oncol. 2015;111(6):663-668. https://doi.org/10.1002/ jso. 23864

58. Ilonzo N, Tsang A, Tsantes S, Estabrook A, Thu Ma AM. Breast reconstruction after mastectomy: a ten-year analysis of trends and immediate postoperative outcomes. Breast. 2017;32:7-12. https:// doi.org/10.1016/j.breast.2016.11.023

59. Flitcroft K, Brennan M, Spillane A. Decisional regret and choice of breast reconstruction following mastectomy for breast cancer: a systematic review. Psychooncology. 2018;27(4):1110-1120. https://doi. org/10.1002/pon.4585

60. İzci F, Sarsanov D, Erdogan Zi, et al. Impact of personality traits, anxiety, depression and hopelessness levels on quality of life in the patients with breast cancer. Eur J Breast Health. 2018;14(2):105-111. https://doi.org/10.5152/ejbh.2018.3724

61. Awan BA, Samargandi OA, Alghamdi HA, et al. The desire to utilize postmastectomy breast reconstruction in Saudi Arabian women. Predictors and barriers. Saudi Med J. 2015;36(3):304-309. https://doi. org/10.15537/smj.2015.3.10688

62. Hamnett KE, Subramanian A. Breast reconstruction in older patients: a literature review of the decision-making process. J Plast Reconstr Aesthet Surg. 2016;69(10):1325-1334. https://doi.org/10.1016/j. bjps.2016.06.003

63. Girotto JA, Schreiber J, Nahabedian MY. Breast reconstruction in the elderly: preserving excellent quality of life. Ann Plast Surg. 2003;50(6):572-578.

64. Sergesketter AR, Pyfer BJ, Phillips BT, Zhao R, Hollenbeck ST. Check the record: remote CT scans for breast flap perforator mapping. J Reconstr Microsurg. 2018;34(7):485-491. https://doi.org/10.1055/s0038-1639578

65. Edwards-Hampton SA, Wedin S. Preoperative psychological assessment of patients seeking weight-loss surgery: identifying challenges and solutions. Psychol Res Behav Manag. 2015;8:263-272. https://doi. org/10.2147/PRBM.S69132

66. Kumnig M, Jowsey-Gregoire S. Preoperative psychological evaluation of transplant patients: challenges and solutions. Transp Res and Risk Manag. 2015;7:35-43. https://doi.org/10.2147/TRRM.S59268

67. Honigman RJ, Jackson AC, Dowling NA. The PreFACE: a preoperative psychosocial screen for elective facial cosmetic surgery and cosmetic dentistry patients. Ann Plast Surg. 2011;66(1):16-23. https://doi.org/ 10.1097/SAP.0b013e3181d50e54

\section{SUPPORTING INFORMATION}

Additional supporting information may be found online in the Supporting Information section at the end of the article.

How to cite this article: Sousa H, Castro S, Abreu J, Pereira MG. A systematic review of factors affecting quality of life after postmastectomy breast reconstruction in women with breast cancer. Psycho-Oncology. 2019;28:2107-2118. https://doi.org/ 10.1002/pon.5206 
Copyright of Psycho-Oncology is the property of John Wiley \& Sons, Inc. and its content may not be copied or emailed to multiple sites or posted to a listserv without the copyright holder's express written permission. However, users may print, download, or email articles for individual use. 